\title{
Penetrating Keratoplasty - Indications and Post Operative Visual Outcome in A South Indian Population
}

\author{
Dr May Saldanha ${ }^{1}$, Dr Norman Mendonca ${ }^{2}$ \\ ${ }^{1}$ (Dept Of Opthalmology, Fr Muller Medical College/ Rajiv Gandhi University of Health Sciences ,India) \\ ${ }_{2}^{2}$ (Dept Of Opthalmology, Fr Muller Medical College/ Rajiv Gandhi University of Health Sciences ,India)
}

\begin{abstract}
Objective:- To discuss the cases who underwent penetrating keratoplasty in terms of indications, post operative visual prognosis and follow up in an south Indian population. Methods:- A retrospective study was done where in patients who underwent penetrating keratoplasty at our hospital from June 2010 to June 2012 were evaluated on the basis of indication for surgery, post-operative visual outcome and graft survival on follow up. Results:-Of a total of 60 patients who underwent keratoplasty, in 30 the indication was pseudophakic bullous keratopathy. Majority of the patients had a significant visual acuity improvement in the immediate post op period. Of these in 14 patients visual acuity deterioration occurred over time due to varied reasons. Conclusion:- Penetrating keratoplasty is a surgery where in the host's diseased cornea is transplanted with donor cornea. Various sociological factors in India make voluntary eye donation a rarity depriving many needy people from undergoing this surgery. In our study we found that despite the significant visual improvement in most patients factors like poor follow up, negligence to instill medications and poor hygiene lead to graft failure in many. Thus emphasis should be laid on the need for eye donation and good hygiene.
\end{abstract}

Keywords - Indications, Keratoplasty, Pentrating, VisualOutcome, South India.

\section{Introduction}

Penetrating keratoplasty is a surgery where in the host's diseased cornea (full thickness) is transplanted with a donor cornea. It is one of the measures to overcome blindness caused due to various conditions affecting the cornea which hamper with the refractive ability, vision clarity and integrity of the cornea.

Keratoplasty was first performed by Zirm in 1905, with very few changes over the past decades and has been increasingly performed in larger numbers with better eye banking facilities. Although newer modalities of keratoplasty have come into play but penetrating keratoplasty still remains the gold standard in corneal conditions involoving full thickness of the cornea. ${ }^{[1]}$

Voluntary eye donation is a necessity for this surgery where in the donor cornea needs to be taken within 6 hours of death and stored in adequate storage media and used for the recipient at the earliest.

Various studies have been done in various western populations with respect to incidence and indications but few attempts have been made to know the same in a south Indian population.

\section{Materials And Methods}

A retrospective study was done where in patients who underwent penetrating keratoplasty at our hospital from June 2010 to June 2012 were evaluated on the basis of indication for surgery, post-operative visual outcome and graft survival on follow up.

Donor corneal button was taken from the cadaver under asepsis within 6 hours of death and placed in MK Medium. Blood was taken from the cadaver and examined for $\mathrm{HIV} / \mathrm{HBsAg} / \mathrm{HCV}$. Keeping the various contraindications for the use of donor corneas (as per eye bank association of America) ${ }^{[2]}$ and the physical donor cornea qualities selected corneas were taken up for surgery.

Within a period of 2-3 days the donor cornea was transplanted on to the recipient corneal bed where the donor cornea was trephined $0.5 \mathrm{~mm}$ more than the recipient cornea and was sutured with $10-0$ ehilon interrupted sutures. Post op patient was started on topical steroids, antibiotics, mydriatics, anti-inflammatory and tear drops and were followed up on regular intervals. Occasional patients had additional antiglaucoma and immunosuppressive agents like topical cyclosporine added.

\section{Results}

Among the patients who underwent surgery 40 (66\%) were males and $20(33 \%)$ were females. Among the patients who underwent surgery $47(78 \%)$ belonged to the urban areas and 13(22\%) belonged to the rural areas.

Among the corneal grafts collected he various indications for surgery in our study population included: 
Table 1: Indications among patients for keratoplasty

\begin{tabular}{lr}
\hline \multicolumn{1}{c}{ INDICATIONS } & NO OF \\
& PATIENTS \\
\hline Pseudophakic Bullous Keratopathy & 30 \\
Sec to Corneal Ulcer (Therapeutic) & \\
& 12 \\
Corneal Opacity & 10 \\
Failed PK & 8 \\
\hline
\end{tabular}

The post op visual acuity was found to improve in majority at the end of 1 week and in the majority of patients was found to be in the range of $1 / 60-6 / 60$.

Table 2: Visual Acuity on Post Op Follow Up Visits

\begin{tabular}{lrrr}
\hline POST OP VISUAL OUTCOME & $<\mathbf{1 / 6 0}$ & $\begin{array}{c}\mathbf{1 / 6 0 -} \\
\mathbf{6 / 6 0}\end{array}$ & $>\mathbf{6 / 6 0}$ \\
\hline 3 days & 32 & 28 & 0 \\
1 week & 8 & 40 & 12 \\
1 month & 4 & 36 & 20 \\
3 months & 14 & 38 & 18 \\
\hline
\end{tabular}

Of the patients 14, had visual deterioration over time, of which 8 had suffered from graft rejection. 10 patients were lost to follow up.

\section{Discussion}

Of the various types of keratoplasties performed, full thickness penetrating keratoplasty is the most commonly performed surgery in those with impaired vision due to full thickness corneal involvement. There are four major factors that cause the cornea its ability to refract light - the apheric contour, irregular surface smoothness, increased stromal thickness and stromal opacity. ${ }^{[3]}$

The importance of this procedure lies in the fact that corneal blindness does not have any other mode of treatment unless the diseased cornea is replaced by a healthy donor cornea which is only possible with voluntary eye donation. In India the recipients requiring eye donation far exceeds the number of voluntary eye donations that are made.

Of a total of 60 patients who underwent keratoplasty in our setup, in 30 the indication was pseudophakic bullous keratopathy. Majority of the patients had a significant visual acuity improvement within 1 week post op. Of these, in 14 patients visual acuity deterioration occurred over time due to improper instillation of drops and lack of hygiene. 10 patients were lost to follow up.

Of a total of 60 patients who underwent keratoplasty in our setup, in 30 the indication was pseudophakic bullous keratopathy. Majority of the patients had a significant visual acuity improvement within 1 week post op. Of these, in 14 patients visual acuity deterioration occurred over time due to improper instillation of drops and lack of hygiene. 10 patients were lost to follow up.

\section{Conclusion}

Penetrating keratoplasty is an important mode of surgery to treat patients with corneal blindness. It is a relatively safe, reliable and inexpensive procedure. It remains the gold standard in treatment for all full thickness involved corneas despite newer advancements in surgery. ${ }^{[4]}$

Emphasis should thus be laid on the need for eye donation, maintenance of a good eye bank register, highlighting on health education and conduction of adequate health camps among the general population with this regard.

Maintainnce of good hygiene, proper instillation of medication and regular follow up post surgery needs to be highly stressed upon. The lack of motivation for eye donation and the poor post op follow up following various ocular diseases makes keratoplasty still a underutlized surgery. Further efforts need to be taken to have better eye banking facilities and various corneal blindness related outreach programmes. ${ }^{[5]}$

none

\section{Acknowledgements}




\section{References}

[1]. J Kanski and B Bowling, Clinical Ophthalmology - A systematic approach( $7^{\text {th }}$ ed), (UK: Elsevier Saunders 2011), 240-244.

[2]. Eye Bank Association of America. Stastical Report on eye banking activity for 2008. (www.restoresight.org)

[3]. Albert \& Jackobiec. Principles and practice of Ophthalmology Vol 2. $2^{\text {nd }}$ edition Chapter 77, 2000,W.B. Saunder's Company .19151936.

[4]. J Reidy, S Bouchard, J Forakis, et Al., American Academy of Ophthalmology - Section 8: External Disease and Cornea , San Fransciso CA, 2010-2011

[5]. L C Dutta, Modern Ophthalmology $1^{\text {st }}$ Volume, L.C. (New Delhi: Jaypee brothers Medical Publishers Ltd. 2008) 